\title{
XXXIV. On the possibility of explaining the phenomena of magnetism by the hypothesis of participation of matter in the motion of the magnetic field
}

\section{B. Rosing}

To cite this article: B. Rosing (1896) XXXIV. On the possibility of explaining the phenomena of magnetism by the hypothesis of participation of matter in the motion of the magnetic field, Philosophical Magazine Series 5, 42:257, 314-332, DOI: $10.1080 / 14786449608620925$

To link to this article: http://dx.doi.org/10.1080/14786449608620925

\section{Published online: 08 May 2009.}

Submit your article to this journal $₫$

Џll Article views: 2

Q View related articles $\longleftarrow$ 
theory. We think that it is indispensable to complete the experiments above deseribed in order to elucidate the various questions which present themselves, and we limit ourselves to a general conclusion that the phenomena observed should be attributed to electric fluxes proceeding from the Hittorf's tube and the objects in its neighbourbood, together with a dielectric polarization; in this sense the similarity of the electric fields of a Hittorf's tube and of an electrified conductor must be admitted; we must therefore take it into account in the study of the electric properties of Röntgen's rays.

April 1896.

XXXIV. On the Possibility of explaining the Phenomena of Magnetism by the Hypothesis of Participation of Matter in the Motion of the Magnetic Field. By B. Rosing, Fellow of the Russian Physico-chemical Society*.

A LL theories of magnetism, whatever their physical founA dations, are founded from the point of view of dynamics on the supposition of the existence of two principal types of physical coordinates; the one fixing the intensity and the distribution of magnetic induction, the other defining the state of the magnetized matter. But the coordinates, as is known, can be in general either of positional or of kinosthenic charactert; $i$.e. they can occur in the expression for the energy of a system either explicitly or only through their differential coefficients. Therefore we may imagine three combinations of our magnetic coordinates, and consequently divide all possible hypotheses on magnetism into three categories. The first category, when both types of coordinates are positional ; Weber's hypothesis, for instance, of Molecular Magnets belongs to it. The other, when the one type is positional and the other kinosthenic; such is Ampère's hypothesis of Molecular Electric Currents in Maxwell's version $\ddagger$ : the latier takes the energy of the electric currents to be kinetic. A third combination is still possible-when both types of coordinates belong to the kinosthenic type, $i$. e. when it is supposed that matter when magnetized is put into the same motion as the surrounding magnetic field. We shall take this third assumption.--Have we the right to consider the magne-

* Communicated by the Author.

+ See J. J. Thomson's 'Applications of Dynamics to Jhysics and Chemistry, p. 10.

I See Maxwell's 'Treatise on Electricity and Magnetism,' 1892, rol. ii. chap. xxii. 
tization of matter as a purely kilietic process, and to explain all magnetic phenomena-such is polarity of paramagnetic and diamagnetic bodies, magnecrystallic force, liysteresis-as simple mechanical consequences of the participation of matter in the motion of rether which takes place in magnetic induction tubes? Of this we have no positive proof; but this hypothesis is attractive by reason of its simplicity, and at the same time does not contain anything improbable. To explain all phenomena by means of the properties of matter in motion and to deduce all laws from the laws of kinetic energy has not this always been what natural philosophy has striven to achieve? The idea of connecting the motion of matter and xther dynamically is also not novel: in this way only was Helmboltz enabled to explain the phenomenon of anomalous dispersion. Magnetism also possesses its own anomalous dispersion : it is paramagnetic polarity of some bodies in relation to diamagnetic polarity of others. And in reality, as we shall see further on, the paramagnetic refraction of lines of magnetic force-to use Faraday's deep and expressive languageis just the same mechanical consequence of absorption of energy. as is the anomalous dispersion of lines of the lightradiation. Diamagnetism of matter, as is known, is explained very easily by admitting the existence of molecular electric currents, excited by the surrounding magnetic field and circulating freely and without resistance on the surface of the particles of matter. Electromagnetism* shows that the energy of a system of spherical currents, referred to one of these currents, is equal to

$$
\mathrm{W}=-\frac{1}{2} \iint \frac{\partial \Omega}{\partial v} \phi d \mathrm{~S}-\Sigma_{\kappa} \iint \frac{\partial \Omega_{\kappa}}{\partial \nu} \phi d \mathrm{~S} ; . . .
$$

where the integrals are extended over the surface of the current in question, $\phi$ is the stream-function, and $\Omega$ and $\Omega^{\star}$ are the magnetic potentials due to the present current and the surrounding ones respectively. The sign $\Sigma$ refers to all the surrounding currents.

Adapting this formula to the case of molecular spherical currents excited by the magnetic field $\mathrm{H}$, and introducing the intensity of magnetization $I$ instead of the magnetic moment of currents, after the transformation the formulit for the energy of molecular currents, referred to unit of volume, will be

* See Burbury's " On the Induction of Electric Currents in Conducting Shells," Phil. T'rans. 1888, p. 302. 


$$
\mathrm{W}=\frac{2 \pi}{3}\left(\frac{2}{\epsilon}+1\right) \mathrm{I}^{2}+\mathrm{IH}, \quad \text {. . . . }
$$

where $\epsilon$ is the aggregate volume of the particles in unit of volume.

At the same time, by applying Lagrange's equation to the expression of the energy of molecular currents, we get the following relation between the magnetization I and the magnetic force $\mathrm{H}:-$

$$
\frac{4 \pi}{3}\left(\frac{2}{\epsilon}+1\right) \mathrm{I}+\mathrm{H}=0 . \quad . \quad . \quad . \quad .
$$

It is evident that this equation can be got by applying Lagrange's equation directly to formula (2) and by regarding I as a velocily.

Formula (3) represents a case of diamagnetism,

$$
I=\frac{-H}{\frac{4 \pi}{3}\left(\frac{2}{\epsilon}+1\right)} .
$$

From it we find the coefficient of magnetization $\kappa$ to be

$$
\kappa=\frac{\mathrm{I}}{\overline{\mathrm{H}}}=-\frac{1}{\frac{4 \pi}{3}\left(\frac{2}{\epsilon}+1\right)} ; \quad . \quad . \quad .
$$

and, lastly, that of the magnetic permeability to be

$$
\mu=\frac{1-\epsilon}{1+\frac{e}{2}} . \text {. . . . . . }
$$

The formula (5) of magnetic permeability is found by assuming the hypothesis of molecular currents, excited by the magnetic field on the surface of particles which are themselves absolutely impermeable. It is remarkable that the same formula can be found by assuming another hypothesis, namely that which takes magnetic induction to be a flux, propagating through media of different conductivity. In reality, as is known, the problem of distribution of magnetic induction in space corresponds exactly with that of electric currents*. But we know from Electrokinematics that the conductivity of a medium consisting of spheres of conductivity $\mu_{2}$, disseminated through a medium of conductivity $\mu_{1}$, is $\dagger$

* See Maxwell's 'Treatise on Electricity and Magnetism,' 1892, vol. ii. p. 54.

+ Ibidem, vol. ii. p. 57. 


$$
\mu=\mu_{1} \frac{2 \mu_{1}+\mu_{2}+2 \epsilon\left(\mu_{2}-\mu_{1}\right)}{2 \mu_{1}+\mu_{2}-\epsilon\left(\mu_{2}-\mu_{1}\right)} . . \text {. . . }
$$

Applying this formula to our case of magnetic induction existing in a space amongst particles of matter absolutely impermeable, we must take $\mu_{1}$ to be equal to 1 , and $\mu_{2}$ equal to 0 . The formula (6) will then take the following form :-

$$
\mu=\frac{1-\epsilon}{1+\frac{\epsilon}{2}}, \text {. . . . . . . }
$$

which is identical with formula (5). It is easy to see from this formula that the coefficient of magnetization $\kappa$ is equal to

$$
\kappa=\frac{-1}{\frac{4 \pi}{3}\left(\frac{2}{\epsilon}+1\right)}, . \quad \text {. . . . }
$$

and $\mathrm{I}$ is connected to $\mathrm{H}$ by the formuli

$$
\frac{4 \pi}{3}\left(\frac{2}{\epsilon}+1\right) \mathrm{I}+\mathrm{H}=0 . \quad \text {. . . . . }
$$

The magnetic energy of unit of volume is, as we know from electromagnetism, expressed in terms of the magnetic induction $\mathrm{B}$ and magnetic force $\mathrm{H}$ by the formula

$$
\mathrm{W}=\frac{1}{8 \pi} \mathrm{BH} \text {. }
$$

By transforming this formula and using the relation (9) we get

$$
\begin{aligned}
W= & \frac{1}{8 \pi} B H=\frac{1}{8 \pi}(H+4 \pi \mathrm{I}) \mathrm{H}=\frac{1}{8 \pi} \mathrm{H}^{2}+\frac{1}{2} \mathrm{IH} \\
& =\frac{1}{8 \pi} \mathrm{H}^{2}+\mathrm{IH}-\frac{1}{2} \mathrm{IH}=\frac{1}{8 \pi} \mathrm{H}^{2}+\mathrm{IH}+\frac{2 \pi}{3}\left(\frac{2}{\epsilon}+1\right) \mathrm{I}^{2} .
\end{aligned}
$$

Here $\frac{1}{8 \pi} \mathrm{H}^{2}$ is the energy of unit volume of magnetic field and the terms $\mathrm{IH}+\frac{2 \pi}{3}\left(\frac{2}{\epsilon}+1\right) \mathrm{I}^{2}$ represent the magnetic energy of the matter. These terms, also formulæ (7), (8), and (9), are, as we see, identical with those found before. Such a coincidence in the results of the hypothesis of Magnetic Flux with those of the hypothesis of Molecular Electric Currents permits of our concluding that a system of spherical currents excited without resistance on the surface of particles of matter is merely 
a mathematical fiction, representing the conditions of the propagation of lines of induction in the space occupied by the particles of matter, namely the conditions of their reflevion at the surface of these particles.

Formula (3) or (8) shows that neither of the hypotheses, when excluding the participation of matter, can explain paramagnetic phenomena: the first, because the magnetic moment of induced currents always appears in a direction opposed to the magnetic field; the second, because the presence of absolutely impermeable matter always lessens the magnetic permeability of space. Consequently we are obliged to introduce a supplementary hypothesis expressing this participation in some way or other.

We introduce it here by supposing that the matter, when in a magnetic field, is itself put into some motion; and consequently, besides the system of coordinates representing molecular electric currents, coordinates also exist which fix this magnetic motion for each particle. As these new coordinates, we suppose, are of kinosthenic character, the new terms, appearing in the magnetic energy of a substance, are of the form

$$
\lambda \mathrm{IJ} \text { and } \frac{1}{2} \nu \mathrm{J}^{2} \text {; }
$$

where $J$ is the vector defining in every point the velocity of magnetic motion of matter, and the coefficients $\lambda$ and $\nu$ depend on the nature of a substance, and denote--the first, $\lambda$, the connexion between the motion of magnetic induction and the magnetic motion of matter, and the second, $\nu$, the inertia of this latter motion.

Thus the magnetic energy of unit of volume will be represented by the following expression:-

$$
\mathrm{W}=\frac{2 \pi}{3}\left(\frac{2}{\epsilon}+1\right) \mathrm{I}^{2}+\mathrm{IH}+\lambda \mathrm{IJ}+\frac{1}{2} \nu J^{2} .
$$

By applying to this expression the principle of Least Action, we obtain Lagrange's equation in a new form : for coordinate I

$$
\frac{d}{d t}\left(\frac{4 \pi}{3}\left(\frac{2}{\epsilon}+1\right) \mathrm{I}+\lambda \mathrm{J}+\mathrm{H}\right)=0, \ldots
$$

for coordinate $\mathrm{J}$

$$
\frac{d}{d t}(\lambda \mathrm{I}+\nu \mathrm{J})=0 . \quad . \quad . \quad . \quad . \quad .
$$

Hence, after integrating and putting the initial conditions $\mathrm{I}=\mathrm{J}=\mathrm{H}=0$, we have 
and therefore

$$
\left.\begin{array}{r}
\frac{4 \pi}{3}\left(\frac{2}{\epsilon}+1\right) I+\lambda J+H=0 \\
\lambda I+\nu J=0
\end{array}\right\},
$$

$$
\mathrm{I}=\frac{-\mathrm{H}}{\frac{4 \pi}{3}\left(\frac{2}{\epsilon}+1\right)-\frac{\lambda^{2}}{\nu}}, \quad . \quad . \quad .
$$

or

$$
\kappa=\frac{-\epsilon}{\frac{4 \pi}{3}(2+\epsilon)-\frac{\epsilon \lambda^{\overline{2}}}{\nu}} . \quad . \quad . \quad .
$$

Formula (15) now contains both cases of magnetization: that of magnetic and that of paramagnetic bodies. In reality, when the coefficient $\lambda$ is sufficiently large and $\boldsymbol{v}$ sufficiently small, $\kappa$ assumes positive values and we enter the sphere of paramagnetism.

From the equations (13) we obtain besides this

$$
\mathrm{J}=-\frac{\lambda}{\nu} \mathrm{I} \text { and } \frac{1}{2} \nu \mathrm{J}^{2}=\frac{1}{2} \frac{\lambda^{2}}{\nu} \mathrm{I}^{2} ;
$$

whence it is evident that-given a comparatively large value to $\lambda$ and small value to $y$-the velocity of magnetic motion of matter and its energy under the same magnetization I are comparatively greater. We conclude from this, that the absorption of energy by motion of matter in paramagnetic. bodies is comparatively greater than in diamagnetic ones, as has been already pointed out, and that in consequence thereof appears that anomalous propagation of the magnetic inductiontubes which is observed in paramagnetic bodies. Besides that, as we already decided to regard the phenomenon of diamagnetism as the reflexion of lines of induction at the surfaces of particles of matter, we must now consider paramagnetism to be also the reflexion of the induction lines, but taking place without change of sign. Thus we find here the same phenomenon of double-signed reflexion with and without change of sign as we also see in other branches of physics, as for instance in the reflexion of waves of light and sound at the surfaces separating media of different nature.

In the case of a crystalline substance the magnetic energy of unit of volume is expressed by a formula which is analogous to formula (10), but the vectors $\mathrm{I}, J, \mathrm{H}$ are replaced here by their components $(\mathrm{A}, \mathrm{B}, \mathrm{C}),(\mathrm{L}, \mathrm{M}, \mathrm{N})$, and $(a, \beta, \gamma)$ respectively. By applying to it Lagrange's equations we get a system of equations : 


$$
\begin{aligned}
& \frac{4 \pi}{3}\left(\frac{2}{\epsilon_{1}}+1\right) \mathrm{A}+\lambda_{x x} \mathrm{~L}+\lambda_{y x} \mathrm{M}+\lambda_{z x} \mathrm{~N}=-\alpha, \\
& \frac{4 \pi}{\ddot{3}}\left(\frac{2}{\epsilon_{2}}+1\right) \mathrm{B}+\lambda_{x y} \mathrm{~L}+\lambda_{y y} \mathrm{M}+\lambda_{z y} \mathrm{~N}=-\beta, \\
& \frac{4 \pi}{3}\left(\frac{2}{\epsilon_{3}}+1\right) \mathrm{C}+\lambda_{x z} \mathrm{~L}+\lambda_{y z} \mathrm{M}+\lambda_{z z} \mathrm{~N}=-\gamma, \\
& \lambda_{x x} \mathrm{~A}+\lambda_{x y} \mathrm{~B}+\lambda_{x z} \mathrm{C}=-\left(v_{x x} \mathrm{~L}+v_{y x} \mathrm{M}+v_{z x} \mathrm{~N}\right), \\
& \lambda_{y x} \mathrm{~A}+\lambda_{y y} \mathrm{~B}+\lambda_{y z} \mathrm{C}=-\left(v_{x y} \mathrm{~L}+v_{y y} \mathrm{M}+v_{z y} \mathrm{~N}\right), \\
& \lambda_{z x} \mathrm{~A}+\lambda_{z y} \mathrm{~B}+\lambda_{z z} \mathrm{C}=-\left(v_{x z} \mathrm{~L}+v_{y z} \mathrm{M}+v_{z z} \mathrm{~N}\right) ;
\end{aligned}
$$

which on excluding $\mathrm{L}, \mathrm{M}, \mathrm{N}$ will be reduced to three equations :

where

$$
\left.\begin{array}{l}
\mathrm{A}=\kappa_{1} \alpha+\kappa_{2} \beta+\kappa_{3} \gamma \\
\mathrm{B}=\kappa_{2}^{\prime} \alpha+\kappa_{2}^{\prime} \beta+\kappa_{3}^{\prime} \gamma \\
\mathrm{C}=\kappa_{1}^{\prime \prime} \alpha+\kappa_{2}^{\prime \prime} \beta+\kappa_{3}^{\prime \prime} \gamma
\end{array}\right\},
$$

$$
\kappa_{1}^{\prime}=\kappa_{2}, \kappa_{1}^{\prime \prime}=\kappa_{3}, \kappa_{2}^{\prime \prime}=\kappa_{3}{ }^{\prime} .
$$

These are, as we know, the fundamental equations for magnetization of a crystalline substance. The coefficients $\kappa_{1}, \kappa_{2}, \kappa_{3}$ ... here may have both positive and negative values depending upon the values $\varepsilon_{1}, \epsilon_{2}, \epsilon_{3}, \lambda_{x x}, \lambda_{x y}, \lambda_{y y}, \ldots v_{x x}, v_{x y}, v_{y y}, \ldots$ for the different axes of the crystal.

Lastly, by introducing into the formula of energy values of $\mathrm{I}, \mathrm{M}, \mathrm{N}$ from the above equations, and by adding $\frac{1}{8 \pi} \mathrm{H}^{2}$, we get

$$
\mathrm{W}=\frac{1}{8 \pi} \mathrm{BH} \cos \mathrm{BH} .
$$

Thus we see that the results found by means of the hypothesis of mechanical participation of matter in phenomena of magnetic induction answer well enough to the fundamental requirements of the theory of magnetism.

However, these suppositions are not sufficient to explain all phenomena of magnetism, for instance the phenomena which take place when iron and such metals are magnetized. The phenomenon of magnetic remanescence forces us, on the other hand, to suppose the existence of magnetic deformations that take place at the magnetic motion of matter, and therefore to accept a new type of coordinates which would define them.

Actually, whatever the magnetic motion of matter, it is, of 
course, a periodic motion round the axis of the vector $\mathrm{J}$. Arising inside matter, it must produce there a kind of pressure, counterbalanced by the elastic forces of matter, and must therefore be accompanied by certain deformations. We suppose that this pressure is kinetic in character, $i$ e., it passes on by collisions from particle to particle.

Now it is easy to prove that such a kinetic pressure must be proportional to the square of the velocity $J$, and that at the moment of the change of this pressure forces of reaction appear which act backward on the vector J.

Actually, the hypothesis of the existence of kinetic pressure produced by the magnetic motion of matter is dyuamically equivalent to supposing that a connexion exists between this motion of matter and the motion arising at its deformations. Therefore, if we denote the coordinates fixing magnetic deformations by the letters $p_{a}, p_{\beta}, \ldots$, then the above hypothesis will mean that terms exist in the formula of Lagrange's function of the following kind :-

$$
\rho_{\alpha} \dot{p}_{\alpha}^{\mathrm{J}}+\rho_{\beta} \dot{p}_{\beta} \mathrm{J}+\ldots, \cdot \cdot \cdot \cdot
$$

whore $\rho_{\alpha}, \rho_{\beta}, \ldots$ are coefficients defining the connexion between the two motions.

Therefore, in Lagrange's equation for the vector J, besides the forces contained already in formula (12), new forces appear and the equation is :-

$$
\begin{array}{r}
\frac{d}{d t}(\lambda \mathrm{I}+\nu \mathrm{J})+\frac{d}{d t}\left(\rho_{a} \dot{p}_{\alpha}+\rho_{\beta} \dot{p}_{\beta}+\ldots\right)-\frac{\partial}{\partial \Theta}\left[\rho_{\alpha} \dot{p}_{\alpha} \mathrm{J}\right. \\
\left.+\rho_{\beta} \dot{p}_{\beta} \mathrm{J}+\ldots\right]=0, .
\end{array}
$$

if we denote by $\Theta$ the coordinate the velocity of which is $\mathrm{J}, i . e$. :

$$
\mathrm{J}=\frac{d \Theta}{d t} \cdot . \quad \cdot \quad \cdot \quad \cdot .
$$

Besides this we shall get the following equations relating to coordinates $p_{\boldsymbol{a}}, p_{\beta}, \ldots$ :-

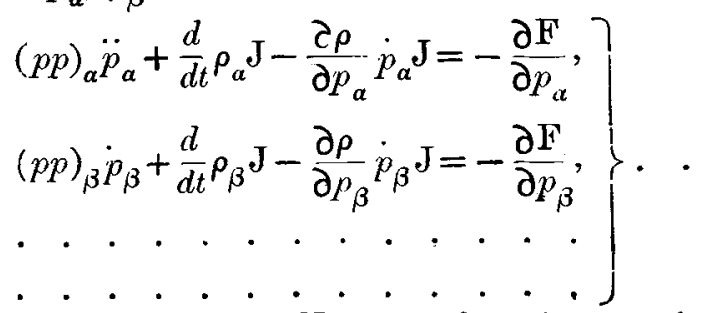

Plit. Mag. S. 5. Vol. 42. No. 257. Oct. 1896. 
Here the terms $(p p)_{\alpha} \ddot{p}_{\alpha},(p p)_{\beta} \ddot{p}_{\beta}, \ldots$ represent forces of inertia for the coordinates $p_{\alpha}, p_{\beta}, \ldots$ and the function $\mathrm{F}$ represents the free energy of deformations according to Helmholtz's theory. It must be observed, however, that we simplified the formulæ by taking the coordinates $p_{\alpha}, p_{\beta}, \ldots$ to be independent of each other, and the functions $\rho_{a}, \rho_{\beta}, \ldots$ to depend only on the coordinates $\Theta$ and $p_{a}, p_{\beta}, \ldots$ respectively. On the supposition of indefinitely slow changes in the coordinates $p_{a}, p_{\beta}, \ldots$ we can neglect the forces of inertia; further, by giving to the equations the form

$$
\begin{aligned}
& \frac{d \rho_{a}}{d \Theta} \mathrm{J}^{2}+\rho_{\alpha} \frac{d \mathrm{~J}}{d t}=-\frac{\partial \mathrm{F}}{\partial p_{\alpha}}, \\
& \frac{d \rho_{\beta}}{d \Theta} \mathrm{J}^{2}+\rho_{\beta} \frac{d \mathrm{~J}}{d t}=-\frac{\partial \mathrm{F}}{\partial \rho_{\beta}},
\end{aligned}
$$

we can neglect the forces $\rho_{\alpha} \frac{d \mathrm{~J}}{d t}, \rho_{\beta} \frac{d \mathrm{~J}}{d t}, \ldots$ on the same grounds. Lastly, putting

$$
\frac{d \rho_{\alpha}}{d \Theta}=-\sigma_{a}, \frac{d \rho_{\beta}}{d \Theta}=-\sigma_{\beta}, \quad . \quad . \quad . \quad .
$$

we get the equations in the case of indefinitely slow changes to be :-

$$
\left.\begin{array}{l}
\sigma_{a} \mathrm{~J}^{2}=\frac{\partial \mathrm{F}}{\partial p_{\alpha}} \\
\sigma_{\beta} \mathrm{J}^{2}=\frac{\partial \mathrm{F}}{\partial p_{\beta}}
\end{array}\right\} \cdot . . . .
$$

which show the equilibrium bctueen the components of pressure proportional to $\mathrm{J}^{2}$ and the corresponding forces of elasticity.

Let us now consider equation (18). After integrating from the initial moment, when $\mathrm{I}=\mathrm{J}=\mathrm{H}=0$, this equation gives :

$$
\lambda \mathrm{I}+\nu \mathrm{J}+\rho_{a} \dot{p}_{\alpha} \mathrm{J}+\rho_{\beta} \dot{p}_{\beta} \mathrm{J}+\ldots-\int_{0}^{t} \frac{\partial \rho_{\alpha}}{\partial \Theta} \dot{p}_{a} \mathrm{~J} d t-\int_{0}^{t} \frac{\partial \rho_{\beta}}{\partial \Theta} \dot{p}_{\beta} \mathrm{J} d t-\ldots=0
$$

Once more assuming the indefinite slowness of change, we shall have for static magnetization : 


$$
\lambda \mathrm{I}+\nu \mathrm{J}-\int_{0}^{p_{\alpha}} \frac{\partial \rho_{\alpha}}{\partial \Theta} \mathrm{J} d p_{\alpha}-\int_{0}^{p_{\boldsymbol{\beta}}} \frac{\partial \rho_{\beta}}{\partial \Theta} \mathrm{J} d p_{\beta}-\ldots=0,
$$

because we can negiect the terms containing $p_{\alpha}, p_{\beta}, \ldots$ and can accept $p_{\alpha}, p_{\beta}, \ldots$ as the independent variables under the signs of integration.

Then, on introducing significations (21), we obtain

$$
\lambda \mathrm{I}+\nu \mathrm{J}+\int_{0}^{p_{a}} \sigma_{\alpha} \mathrm{J} d p_{a}+\int_{0}^{p_{\beta}} \sigma_{\beta} J d p_{\beta}+\ldots=0 .
$$

Thus we have the following system of equations in case of static magnetization :- Lagrange's equation (11) for the vector I, which remains unchanged under the new suppositions, equation (23), and the system of equations (22):

$$
\begin{aligned}
& \frac{d}{d t}\left[\frac{4 \pi}{3}\left(\frac{2}{\epsilon}+1\right) \mathrm{I}+\lambda \mathrm{J}+\mathrm{H}\right]=0, \\
& \lambda \mathrm{I}+\nu \mathrm{J}+\int_{0}^{p_{a}} \sigma_{\alpha} \mathrm{J} d p_{\alpha}+\int_{0}^{p_{\beta}} \sigma_{\beta} \mathrm{J} d p_{\beta}+\ldots=0, \\
& \sigma_{\alpha} \mathrm{J}^{2}=\frac{\partial \mathrm{F}}{\partial p_{\alpha}}, \sigma_{\beta} \mathrm{J}^{2}=\frac{\partial \mathrm{F}}{\partial p_{\beta}}, \ldots
\end{aligned}
$$

Let us integrate the first of these equations and introduce the $J$ from it into the second term of the second equation. Then, by making use of quantities $\sigma_{a} \mathrm{~J}, \sigma_{\beta} \mathrm{J}, \ldots$ from the third equation, and by introducing all th:se quantities under the signs of integration in the second equation, after denoting the sum

by $\delta \mathrm{F}$,

$$
\frac{\partial \mathrm{F}}{\partial p_{a}} d p_{a}+\frac{\partial \mathrm{F}}{\partial p_{\beta}} d p_{\beta}+, \ldots
$$

$$
\delta \mathrm{F}=\frac{\partial \mathrm{F}}{\partial p_{a}} d p_{a}+\frac{\partial \mathrm{F}}{\partial p_{\beta}} d p_{\beta}+\ldots, \cdot . .
$$

the system of our equations will be transformed into the following system :

$$
\begin{gathered}
\frac{4 \pi}{3}\left(\frac{2}{\epsilon}+1\right) \mathrm{I}+\lambda \mathrm{J}+\mathrm{H}=0, . \\
\mathrm{I}=\kappa \mathrm{H}-\kappa \frac{\lambda}{v} \int \frac{\delta \mathrm{F}}{\mathrm{J}}, . . \\
\sigma_{\alpha} \mathrm{J}^{2}=\frac{\partial \mathrm{F}}{\partial p_{\alpha}}, \sigma_{\beta} \mathrm{J}^{2}=\frac{\partial \mathrm{F}}{\partial p_{\beta}}, . .
\end{gathered}
$$


Here the coefficient $\kappa$ is expressed by the above formula (15),

$$
\kappa=\frac{-\epsilon}{\frac{4 \pi}{3}(2+\epsilon)-\frac{\epsilon \lambda^{2}}{\nu}}
$$

and $\delta \mathrm{F}$ is defined by equation (24).

This system of equations represents the general conditions of static magnetization on the supposition of the existence of magnetic pressure and magnetic deformations. We will proceed to examine these formulæ.

Formula

$$
\mathrm{I}=\kappa \mathrm{H}-\kappa \frac{\lambda}{v} \int \frac{\delta \mathrm{F}}{\mathrm{J}}
$$

shows that the process of magnetization is composed of two processes, one, expressed by term $\kappa \mathrm{H}$, is a process which can be completely reversible and which represents, as we have already had occasion to see, a simple reflexion of inductionlines from matter; the other process is expressed by the term

$$
-\kappa \frac{\lambda}{\nu} \int \frac{\delta \mathrm{F}}{\mathrm{J}}
$$

and arises in consequence of absorption of energy by the magnetic deformations. The value of this term wholly depends on the free energy $\mathrm{F}$ of deformations. It is in the nature of these deformations that the explanation of all the complex and intricate phenomena which appear in the magnetization of iron must be sought.

It is evident from equation (25), that in a paramagnetic substance-given $I$ and $H$ positive-the vector $J$ has the opposite sign to the coefficient $\lambda$, and $\kappa$ having a positive value, the product $-\kappa \frac{\lambda}{\nu} \frac{1}{J}$ is greater than nil. On the other hand, when $I$ and $H$ are increased, and consequently $J$ too, the deformations likewise increase, and with them F. Therefore the integral

$$
-\kappa \frac{\lambda}{\nu} \int \frac{\delta \mathrm{F}}{\mathrm{J}}
$$

taken from the beginning of magnetization $I=J=H=0$ is a positive quantity.

Thus, owing to the absorption of energy in a paramagnetic substance, magnetization increases.

To show this still more clearly, we will put the equations 
into differential form. Let us suppose that the process of magnetization takes place at constant temperature. In this case the free energy $\mathrm{F}$ is a function only of the coordinates $p_{\alpha}, p_{\beta}, \ldots$ But these coordinates can be expressed by the system of equations (27) as a function of $\mathrm{J}^{2}$. Therefore the energy $F$ may also be considered as a function of the same quantity $\mathrm{J}^{2}$. In this way we have

$$
\delta \mathrm{F}=\frac{\partial \mathrm{F}}{\partial J^{2}} d \mathrm{~J}^{2} . \quad . \quad . \quad . \quad . \quad .
$$

If we now differentiate the equation (26), considering $\mathrm{H}$ as an independent variable and using the formula (28), we have

$$
\frac{d \mathrm{~J}}{d \mathrm{H}}=1-\frac{2 \kappa \lambda}{\nu} \frac{\partial \mathrm{F}}{\partial J^{2}} \frac{d \mathrm{~J}}{d \mathrm{H}}
$$

let us replace $\frac{d \mathrm{~J}}{d \mathrm{H}}$ by its expression from equation (25);

$$
\frac{d \mathrm{~J}}{d \mathrm{H}}=-\frac{1}{\lambda}\left[\frac{4 \pi}{3}\left(\frac{2}{\epsilon}+1\right) \frac{d \mathrm{I}}{d \mathrm{H}}+1\right]
$$

we have, lastly,

$$
\frac{d \mathrm{I}}{d \mathrm{H}}=\kappa \frac{1+\frac{2}{\nu} \frac{\partial \mathrm{F}}{\partial J^{2}}}{1-\frac{2 \kappa}{\nu} \frac{4 \pi}{3}\left(\frac{2}{\epsilon}+1\right) \frac{\partial \mathrm{F}^{\mathrm{H}}}{\partial J^{2}}} . .
$$

This is the differential equation of static and isothermic magnetization. Here, as has been said above, $\frac{\partial F}{\partial J^{2}}$ must be considered as a function of $\mathrm{J}^{2}$, where $\mathrm{J}$, in its turn, is expressed in terms of $I$ and $H$ by help of equation (25),

$$
\frac{4 \pi}{3}\left(\frac{2}{\epsilon}+1\right) I+\lambda J+H=0 .
$$

The form of this function of $F$ is defined by the way in which $F$ depends on $p_{\alpha}, p_{\beta} \ldots$ as shown in equations (27). Actually, by differentiating the equations (27) at a constant temperature,

$$
\sigma_{\alpha} d J^{2}=\frac{\partial^{2} \mathrm{~F}}{\partial p_{\alpha}^{2}} d p_{a}, \quad \sigma_{\beta} d J^{2}=\frac{\partial^{2} \mathrm{~F}}{\partial p_{\beta}^{2}} d p_{\beta}, \ldots,
$$

and by introducing the quantities $d p_{a}, d p_{\beta}, \ldots$ from here in the expression (24) for $\delta \vec{F}$, we shall have 


$$
\delta \mathrm{F}=\left\{\sigma_{\alpha} \frac{\frac{\partial \mathrm{F}}{\partial p_{\alpha}}}{\frac{\partial^{2} \mathrm{~F}}{\partial p_{\alpha}^{2}}}+\sigma_{\beta} \frac{\frac{\partial \mathrm{F}}{\partial p_{\beta}}}{\frac{\partial^{2} \mathrm{~F}}{\partial p_{\beta}^{2}}}+\ldots\right\} d \mathrm{~J}^{2}
$$

whence, by comparing this equation with (28), we have

$$
\frac{\partial F}{\partial J^{2}}=\left\{\sigma_{\alpha} \frac{\frac{\partial F}{p_{\alpha} F}}{\partial p_{\alpha}^{2}}+\sigma_{\beta} \frac{\frac{\partial F}{\partial p_{\beta}}}{\frac{\partial^{2} F}{\partial p_{\beta}^{2}}}+\ldots\right\},
$$

or, more simply, taking $\frac{\partial \mathrm{F}}{\partial p_{\alpha}}=\mathrm{P}_{\alpha}, \frac{\partial \mathrm{F}}{\partial p_{\beta}}=\mathrm{P}_{\beta}, \ldots$;

$$
\frac{\partial \mathrm{F}}{\partial J^{2}}=\left\{\sigma_{\alpha} \frac{d p_{\alpha}}{d \log \mathrm{P}_{\alpha}}+\sigma_{\beta} \frac{d p_{\beta}}{d \log \mathrm{P}_{\beta}}+\ldots\right\}_{\theta=\text { const. }} \text {. }
$$

where $\theta$ is the absolute temperature, and $\mathrm{P}_{\alpha}, \mathrm{P}_{\beta}, \ldots$ are supposed for simplicity to be only functions of this temperature and of the coordinates $p_{a}, p_{\beta}, \ldots$ respectively.

It is by this equation, in conjunction with equations (25), (27), and (29), that the process of maguetization is completely delined.

First of all, it is to be seen from equation (30) that $\frac{\partial F}{\partial J^{2}}$ is a positive quantity, as $\frac{d p_{\alpha}}{d \log \overline{\mathbf{P}}_{\alpha}}, \frac{d p_{\beta}}{d \log \mathbf{P}_{\beta}}, \ldots$ are, in general, greater than nil.

Therefore, as shown by equation (29), $\frac{d \mathrm{I}}{d \mathrm{H}}$ is for a paramagnetic substance always larger than $\kappa$ if only

$$
\frac{\partial \mathrm{F}}{\partial J^{2}}<\frac{\nu}{2 \kappa} \frac{3}{4 \pi\left(\frac{2}{\epsilon}+1\right)}
$$

When $\frac{\partial \mathrm{F}}{\partial \mathrm{J}^{2}}=\frac{\nu}{2 \kappa} \frac{3}{4 \pi\left(\frac{2}{\epsilon}+1\right)}, \frac{d \mathrm{I}}{d \mathrm{H}}$ is infinitely great, and, lastly, if with increasing of $\mathrm{H}$, and therefore of $\mathrm{J}^{2}, \frac{\partial F}{\partial J^{2}}$ becomes $>\frac{\nu}{2 \kappa} \frac{3}{4 \pi\left(\frac{2}{\epsilon}+1\right)}$, the equation cannot give real solutions, and statical magnetization is impossible.

[In the latter case, as is easily prored, the process of magnetization represents a kind of free motion at a constant magnetic force. Nevertheless, the equations of this process are 
included in the general equations, and can be shown as follows :-

$$
\begin{gathered}
\mathrm{H}=\text { const., } \frac{4 \pi}{3}\left(\frac{2}{\epsilon}+1\right) \mathrm{I}+\lambda \mathrm{J}+\mathrm{H}=0, \\
\sigma_{\alpha} \mathrm{J}^{2} \frac{d p_{\alpha}}{d \mathrm{~J}^{2}}+\sigma_{\beta} \mathrm{J}^{2} \frac{d p_{\beta}}{d \mathrm{~J}^{2}}+\ldots=\frac{\nu}{2 \kappa} \frac{3}{\frac{4 \pi}{3}\left(\frac{2}{\epsilon}+1\right)}, \\
(p p)_{\alpha} \ddot{p}_{\alpha}+\mathrm{G}_{\alpha} \dot{p}_{\alpha}+\sigma_{\alpha} \mathrm{J}^{2}=\frac{\partial \mathrm{F}}{\partial p_{\alpha}} \\
(p p)_{\beta p_{\beta}}+\mathrm{G}_{\beta} \dot{p}_{\beta}+\sigma_{\beta} \mathrm{J}^{2}=\frac{\partial \mathrm{F}}{\partial p_{\beta}} .
\end{gathered}
$$

The new terms introduced in the equations appertaining to the coordinates $p_{\alpha}, p_{\beta}, \ldots ;(p p)_{\alpha} \ddot{p}_{\alpha}+G_{\alpha} \dot{p}_{\alpha},(p p)_{\beta} p_{\beta}+, G_{\beta} \dot{p}_{\beta}, \ldots$ represent the forces of inertia and viscosity respectively. It is clear that in the carve of magnetization showing the dependence of magnetization $I$ on the magnetic force $H$, this process is shown by the straight-lined parts of it parallel to the axis of magnetization.]

The same equation shows, further, that at the commencement of magnetization $\frac{d \mathrm{I}}{d \mathrm{H}}$ must be equal to $\kappa$, because in these conditions $\frac{d p}{d \log \mathrm{P}}=0$; and therefore $\frac{\partial \mathrm{F}}{\partial J^{2}}=0$, if only $\frac{d p}{d \overline{\mathrm{P}}}$ is not $\infty$, which can happen only in particular cases; for instance, near the temperature of recalescence. This is the least value that $\frac{d \mathrm{I}}{d \mathrm{H}}$ can have; it is not great, and if the deformations were not existing, the magnetization of iron would not differ much from the magnetization of other paramagnetic bodies. But that in consequence of these deformations, $\frac{\partial \mathrm{F}}{\partial J^{2}}$ increases very rapidly, and $\frac{d \mathrm{I}}{d \mathrm{H}}$ increases with it. The quantity $\frac{d \mathrm{~F}}{d J^{2}}$ exists as long as the deformations change; when the deformations cease to change, the differential coefficient $\frac{d \mathrm{I}}{d \mathrm{H}}$ again takes sinall values. This ceasing of change of deformations must be therefore supposed in order to explain so-called magnetic saturation. But if we take into consideration that magnetic deformations most likely do not represent 
changes of volume or form, but of the structure of natterlike that which takes place in recalescence, as is most probable,-then there is nothing impossible in this hypothesis. The explanation of another very important phenomenonmagnetic hysteresis-is based on the same phenomenon of magnetic deformations; we must only suppose that the same hysteresis exists in the changing of magnetic deformations.

It is also interesting to see to what our hypothesis could lead in the cise of a diamagnetic substance. In this last case, when $\kappa<0, i$. $e$. the reflexion of magnetic induction from the particles of matter takes place with the changing of the sign, the absorption of energy by deformations gives rise to a quite opposite effect. Formula (29) shows that when $\kappa<0$, the absolute value of $\frac{d \mathrm{I}}{d \mathrm{H}}$ cannot be more than $\kappa$,

$$
\kappa=\frac{-1}{\frac{4 \pi}{3}\left(\frac{2}{\epsilon}+1\right)-\frac{\lambda^{2}}{v}}
$$

because, as it is very easy to see from this formula, the following inequality always holds here,

$$
-\kappa \frac{4 \pi}{3}\left(\frac{2}{\epsilon}+1\right)>1 \text {. }
$$

Further, the same formula shows that $\frac{d l}{d \hat{H}}$ is lessened always with increasing of $\frac{\partial F}{\partial J^{2}}$; and therefore in all such cases, when magnetic deformations increase the most the differential coefficient $\frac{d \mathrm{I}}{d \mathrm{H}}$ falls to its least value.

Owing to this, when we assume the character of the change of deformations in a diamagnetic body to be the same as in a paramagnetic one, we shall have for a diamagnetic substance a curve of magnetization of quite a different form, namely, like the line $\mathrm{OMN}$ shown in the figure. Similarly, when the magnetizing force is decreased, the deformations change more slowly, in consequence of hysteresis, than when it is increased, and the return curve $\mathrm{NPQ}$ descends below the curve $O \mathrm{MN}$ and intersects the axis of $\mathrm{H}$ before $\mathrm{O}$ in the point $\mathrm{P}$. Again, with a further decrease of $\mathrm{H}$, magnetization becomes positive, and the body at $\mathrm{H}=0$ has a paramagnelic residual. This, as is known, was observed by Messrs. QuintusIcilius, Tumlirtz, Lodge, and others.

Besides the differential equation of magnetization, it is 
in the Motion of the Magnetic Field.

important to examine the signification of the integral

$$
-\int \mathrm{I} d \mathrm{H}
$$

which represents, as is known, the area of a closed curve of magnetization.

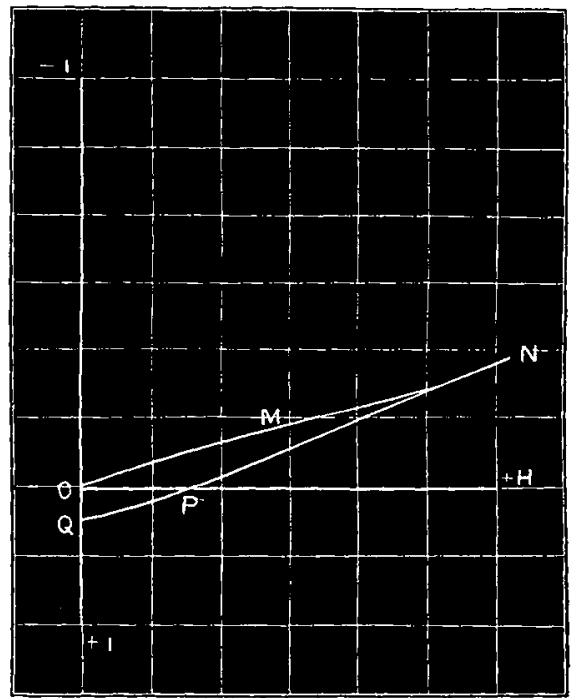

If we write the equation (11) in the form

$$
\frac{4 \pi}{3}\left(\frac{2}{\epsilon}+1\right) \frac{d \mathrm{I}}{d t}+\lambda \frac{d \mathrm{~J}}{d t}=-\frac{d \mathrm{H}}{d t},
$$

the term $-\frac{d \mathrm{H}}{d t}$ will represent the force by which the exterior magnetic field acts on the coordinate, the velocity of which is I. In the time dt it is obvious that this force will perform the work

$$
d \mathrm{~W}=-\frac{d \mathrm{H}}{d t} \cdot \mathrm{I} d t=-\mathrm{I} d \mathrm{H} .
$$

Therefore during the complete cycle of magnetization the magnetic field will perform the work

$$
\mathrm{W}=-\int \mathrm{I} d \mathrm{H}
$$

where the integral is extended along the curve of magnetization.

On the other hand, if we differentiate equation (26), multiply it by $J$, and substitute for $J$ its expression from (25), then we 
shall have

and therefore

$$
J d \mathrm{I}-J \kappa d \mathrm{H}=-\kappa \frac{\lambda}{y} \delta \mathrm{F}
$$

$$
\begin{gathered}
-\frac{4 \pi}{3}\left(\frac{2}{\epsilon}+1\right) \mathrm{I} d \mathrm{I}-\mathrm{H} d \mathrm{I}+\frac{4 \pi}{3}\left(\frac{2}{\epsilon}+1\right) \kappa \mathrm{I} d \mathrm{H}+\kappa \mathrm{H} d \mathrm{H} \\
=-\kappa \frac{\lambda^{2}}{\nu} \delta \mathrm{F} .
\end{gathered}
$$

After integrating along the closed curve, we have, in consequence of equalities which hold in this case,

the equality

$$
\int \mathrm{H} d \mathrm{H}=\int \mathrm{I} d \mathrm{I}=0, \quad \int \mathrm{H} d \mathrm{I}=-\int \mathrm{I} d \mathrm{H},
$$

$$
-\left(1+\frac{4 \pi}{3}\left(\frac{2}{\epsilon}+1\right) \kappa\right) \int \mathrm{I} d \mathrm{H}=\kappa \frac{\lambda}{\nu} \int \delta \mathrm{F} .
$$

But formula (15) shows that

$$
1+\frac{4 \pi}{3}\left(\frac{2}{\epsilon}+1\right) \kappa=\kappa \frac{\lambda^{2}}{\nu}
$$

consequently

$$
-\int \mathrm{I} d \mathrm{H}=\int \delta \mathrm{F} \text {. }
$$

Further,

$$
d \mathrm{~F}=\delta \mathrm{F}-\frac{\partial \mathrm{F}}{\partial \theta} d \theta
$$

where $\theta$ is the absolnte temperature.

But the theory of Free Energy tells us that

$$
d \mathrm{~F}^{\prime}=d \mathrm{U}-d \mathrm{~S} \theta \text {, }
$$

where $U$ is the complete energy of the body and $S \theta$ its bound energy.

Therefore

$\int \delta \mathrm{F}=\int d \mathrm{U}-\int d \mathrm{~S} \theta-\int \frac{\partial \mathrm{F}}{\partial \theta} d \theta=\int d \mathrm{U}-\int \theta d \mathrm{~S}=\int d \mathrm{U}-\mathrm{A} \int d \mathrm{Q}$, because $\frac{\partial F}{\partial \theta}=\mathrm{S}, i$. e. the entropy, and $\theta d \mathrm{~S}=\mathrm{A} d \mathrm{Q}$, where $\mathrm{Q}$ is the quantity of heat supplied to the body and $A$ the mechanical equivalent of heat.

In the case of an isothermic process, when the body on completing the cycle arrives at the initial conditions, we have

and therefore

$$
\int d \mathrm{U}=0 \text {; }
$$

$$
\mathrm{W}=-\int \mathrm{I} d \mathrm{H}=\int \delta \mathrm{F}=-\mathrm{A} \mathrm{Q} \text {. }
$$


But in the case of deformations produced by exterior forces $\int \delta \mathrm{F}$ is, in natural conditions, either nil or a positive quantity. Therefore $\int \mathrm{I} d \mathrm{H}$ is a negative quantity, and magnetization proceeds along the curve in a direction opposite to that in which clock-hands move; and the work performed by the magnetic field is positive, and leaves the body in the form of heat.

Thus, in conclusion, we see that, from the point of view of the hypothesis which considers the magnetic induction as $a$ kind of motion of ather communicated also to matter, we can explain the phenomenon of magnetization as a simple reflexion of tubes of induction from matter, where this reflexion appears with or without a change of sign, with this or that intensity, depending on the forces of reaction of matter which exist at the moment of reflexion. These forces of reaction depend, in their turn, firstly, on the inertia of matter in relation to the magnetic motion and on the coefficient of connexion of æther and matter, which determine the sign of reflexion; secondly, also on the elastic forces in these substances in which magnetic motion is accompanied by magnetic deformations. In those bodies which have a comparatively large magnetic inertia, the reflexion takes place with change of sign and the intensity of reflected induction is greater, the less the inertia and other forces of resistance; this is a normal magnetization, and these bodies are so-called diamagnetic bodies. In others where the inertia is less than a certain quantity, the reflexion takes place without change of sign, and every increase of resistance of matter is accompanied by increase of reflexion. These are so-called paramagnetic bodies.

By following in this way the hypothesis treated of here, one can reduce to the same principles the phenomena observed at rapid and alternate magnetization and find an answer to the very important question of the existence of magnetic inertia and viscosity, and of their rôle in the magnetic circuit. This question, however, necessitates very minute study, and wo shall return to it in the future. At present we will only remark that the results to which this hypothesis brings us are directly opposed to those which are arrived at from the point of view of Weber-Ewing's theory. In opposition to this theory, the magnetization of iron here rises with the increase of resistance offered by the forces of inertia or viscosity. In this way inertia, when the magnetizing current is closed rapidly, must involve a greater magnetization; the same is to be said concerning viscosity; on the other hand, when magnetizing current is alternate, viscosity alone increases it; whereas inertia lessens it; and as in all probability, owing to 
the rearrangement of particles, the viscosity steadily lessens, this entitles us to suppose that with an alternate magnetization the maximum of it does not reach that quantity attained by $a$ static process. And certainly the most recent observations on the exhaustion of iron in transformers seem to corroborate this. From the point of view of the present hypothesis, the exhaustion of iron is nothing but the rearrangement of its particles, in consequence of which the magnetization is performed with a smaller absorption of energy, and therefore calls for a less pronounced magnetic effect.

Physico-chemical Society.

The University, St. Petersburg, Russia, June $15,1896$.

XXXV. Microscopic Vision. By G. Johnstone Stonex, M.A., I).Sc., F.R.S.*

\section{Part I.-Fundamental Principles $†$.}

1. VISION, whether by the naked eye or with the assistance of optical instruments, may be studied in many ways ; since a correct investigation may start from any one of the innumerable possible resolutions of the disturbance which exists throughout the ether in front of and close to the object. But two only of these will be here considered, viz.: that most obrious resolution in which the æther in front of the object is regarded as traversed by undulations of hemisplierical wares emanating from each physical point of the surface of the object, and that other equally general but less obvious resolution of the disturbance in this portion of the æther into undulations of uniform plane waves transmitted forwards in all or some directions from the whole extent of the objective field.

2. The first of these modes of resolution--that into spberical waves-is the foundation of Airy's method of studying the images formed by telescopes, in which the image is regarded as arising from the overlapping and interference of the spurious disks with attendant rings which in the image take the place of points on the object. This method has on this account been sometimes called the Spurious Disk Theory. The second mode of resolution-that into plane waves-is the foundation of Abbe's method of studying the images formed

* Communicated by the Author.

$\uparrow$ Part II. deals with the application of these principles to the microscope as at present made. 